OPEN ACCESS

Edited by:

Ehsan Ahmadpour,

Tabriz University of Medical Sciences,

Iran

Reviewed by:

Apichat Vitta

Naresuan University, Thailand

Fabiana De Paula, Hospital das Clinicas da FMUSP,

Brazil

*Correspondence:

Meysam Sharifdini sharifdini@gums.ac.ir; sharifdini5@gmail.com

Specialty section:

This article was submitted to Clinical Microbiology, a section of the journal

Frontiers in Cellular and Infection Microbiology

Received: 16 August 2021 Accepted: 20 September 2021 Published: 13 October 2021

Citation:

Pandi $M$, Sharifdini $M$, Ashrafi K, Atrkar Roushan Z, Rahmati B and Haijpour N (2021)

Comparison of Molecular and Parasitological Methods for Diagnosis of Human Trichostrongylosis. Front. Cell. Infect. Microbiol. 11:759396. doi: 10.3389/fcimb.2021.759396

\section{Comparison of Molecular and Parasitological Methods for Diagnosis of Human Trichostrongylosis}

\author{
Mehdi Pandi ${ }^{1}$, Meysam Sharifdini ${ }^{1 *}$, Keyhan Ashrafi ${ }^{1}$, Zahra Atrkar Roushan ${ }^{2}$, \\ Behnaz Rahmati ${ }^{1}$ and Nayereh Hajipour ${ }^{3}$ \\ ${ }^{1}$ Department of Medical Parasitology and Mycology, School of Medicine, Guilan University of Medical Sciences, Rasht, Iran, \\ ${ }^{2}$ Department of Biostatistics, School of Medicine, Guilan University of Medical Sciences, Rasht, Iran, ${ }^{3}$ Department of \\ Microbiology, School of Medicine, Guilan University of Medical Sciences, Rasht, Iran
}

Human trichostrongyliasis is a zoonotic disease that is prevalent among rural populations in some countries. This study was performed to evaluate various parasitological methods and polymerase chain reaction (PCR) for the diagnosis of human trichostrongyliasis. A total of 206 fresh stool samples were collected from residents of endemic villages of Northern Iran. All samples were examined using conventional parasitological methods, including wet mount, formalin ethyl acetate concentration (FEAC), agar plate culture (APC), Harada-Mori culture (HMC), and Willis, along with the PCR technique. Among the total of 206 individuals examined, 72 people (35\%) were found infected with Trichostrongylus species using combined parasitological methods. By considering the combined results of parasitological methods as the diagnostic gold standard, the Willis technique had a sensitivity of $91.7 \%$ compared with 52.8\% for the APC, 40.3\% for the HMC, 37.5\% for FEAC, and 5.6\% for the wet mount technique. The diagnostic specificity of all the parasitological methods was 100\%. Furthermore, the PCR method detected Trichostrongylus spp. DNA in 79 fecal samples (38.3\%) with a sensitivity of $97.2 \%$ and a specificity of $93.3 \%$. According to the current findings, the Willis method was more sensitive than are the other parasitological methods in the diagnosis of human trichostrongyliasis. However, the PCR assay was more sensitive and more reliable in the detection of human trichostrongyliasis in comparison with the parasitological methods.

Keywords: human trichostrongyliasis, wet mount, Harada-Mori culture, Willis, agar plate culture, formalin ethyl acetate concentration, PCR

\section{INTRODUCTION}

Nematodes of the genus Trichostrongylus are primarily parasites of herbivorous animals with a worldwide distribution. Ruminants are considered the most important reservoir for human trichostrongylosis (Ghadirian and Arfaa, 1975). Human infections associated with Trichostrongylus species have been reported sporadically from various countries of the Middle and the Far East, Africa, South America, Europe, and Oceania, with the highest prevalence rates 
reported in Iran (Ghadirian and Arfaa, 1975; Cancrini et al., 1982; Boreham et al., 1995; Lattes et al., 2011; Sato et al., 2011; Wall et al., 2011; Phosuk et al., 2013; Watthanakulpanich et al., 2013). In recent decades, a sharply decreasing trend was observed in the prevalence of most human soil-transmitted helminths (STHs) in Iran (Rokni, 2008; Sharifdini et al., 2017b; Sharifdini et al., 2020). However, recent epidemiological studies have demonstrated that trichostrongylosis is still a common helminth infection in humans in some parts of Iran, which results from its ability of zoonotic transmission to humans (Ashrafi et al., 2015; Gholami et al., 2015; Sharifdini et al., 2017a; Sharifdini et al., 2017c; Ashrafi et al., 2020).

Twelve valid species of Trichostrongylus have been detected from humans in various areas of the world, nine of which were only reported from Iran (Ghadirian et al., 1974; Ghadirian and Arfaa, 1975; Ghadirian, 1977; Sharifdini et al., 2017a). Within the past decades, in most parts of Iran, the predominant species of Trichostrongylus in humans were Trichostrongylus orientalis and Trichostrongylus colubriformis (Ghadirian and Arfaa, 1975). Agricultural use of night soil as fertilizer was an important reason for the high prevalence of $T$. orientalis in these regions because the transmission of this species primarily occurs from human to human (Ghadirian and Arfaa, 1975). At present, the predominant species is T. colubriformis because of its high prevalence in domestic animals and its high zoonotic potential (Gholami et al., 2015; Sharifdini et al., 2017a; Sharifdini et al., 2017c).

The transmission route of human infections is mainly through the ingestion of vegetables contaminated with filariform larvae (Roberts and Janovy, 2012). Although trichostrongylosis is generally considered asymptomatic and the only present finding is low-grade peripheral eosinophilia, heavy infections may be followed by abdominal discomfort, diarrhea, nausea, anorexia, weakness, flatulence, dizziness, generalized fatigue or malaise, and mild anemia (Ghanbarzadeh et al., 2019).

Definitive diagnosis of trichostrongylosis depends on observing the characteristic eggs in stool samples or finding the larva in fecal cultures (Roberts and Janovy, 2012). It should be noted that Trichostrongylus eggs may be mistaken with those of hookworms (Sato et al., 2011). Additionally, Trichostrongylus larvae are relatively similar to those of hookworms and Strongyloides stercoralis, which may be difficult to distinguish clearly (Roberts and Janovy, 2012). Although there are several parasitological methods for the detection of Trichostrongylus infection, there are limited studies that show comparisons of their sensitivity and specificity (Najmi et al., 2017; Saraei et al., 2019). Recently, only a few studies applied polymerase chain reaction (PCR)-based techniques for specific detection of Trichostrongylus spp. in human fecal samples (Gholami et al., 2015; Sharifdini et al., 2017c; Perandin et al., 2018). However, these studies had small sample sizes and insufficient power to

Abbreviations: FEAC, formalin ethyl acetate concentration; APC, agar plate culture; HMC, Harada-Mori culture; STHs, soil-transmitted helminths; ITS, internal transcribed spacer; PCR, polymerase chain reaction. evaluate their efficacy. In this study, we compared several parasitological methods along with conventional PCR for the diagnosis of Trichostrongylus infection in human fecal samples.

\section{METHODS}

\section{Study Area and Sample Size Determination}

The study area comprises highly endemic villages of trichostrongylosis within the Fouman District in Guilan Province, Northern Iran. The sample size was calculated based on the prevalence of Trichostrongylus in the region using the following formula: $Z^{2}$ se $(1-\mathrm{se}) / d^{2} \times$ prev, where prev is the prevalence of Trichostrongylus, se is sensitivity, $d$ is the precision of the estimate, and $Z$ is the standard score corresponding to 1.96. The prevalence rate of Trichostrongylus in the study area based on a pilot study and unpublished data was about 36\%. For the calculation, a $95 \%$ sensitivity and a $5 \%$ precision of estimate were used. This gave a sample size of 197. To minimize errors arising from the likelihood of non-compliance, $5 \%$ of the sample size was added, giving a final sample size of about 206 .

\section{Sample Collection}

A total of 206 fresh stool samples were collected from the residents of the villages from June to October 2020. Fecal samples were transferred immediately after collection to the Department of Parasitology and Mycology, Guilan University of Medical Sciences.

\section{Parasitological Methods}

All stool samples were examined using the wet mount, formalin ethyl acetate concentration (FEAC), agar plate culture (APC), Harada-Mori culture (HMC), and Willis techniques.

The APC method was applied for the detection of Trichostrongylus spp. larvae. In brief, 3-4 g of each fecal sample was placed on nutrient agar culture. After incubation for $3-5$ days at room temperature $\left(25-35^{\circ} \mathrm{C}\right)$, the plates were examined under a stereomicroscope for the presence of moving larvae or their tracks. In order to collect the larvae, the surface of the positive agar plates was washed out by lukewarm phosphatebuffered saline (PBS) solution. Larvae of Trichostrongylus species were identified from other probable intestinal nematodes, such as S. stercoralis and hookworms, based on morphological characteristics (Sharifdini et al., 2017a).

In the HMC method, approximately $2 \mathrm{~g}$ of each fresh stool sample was smeared on a folded strip of filter paper. After adding up to $5 \mathrm{ml}$ of distilled water into a $15-\mathrm{ml}$ falcon tube, the strip containing the fecal sample was placed into the tube and stored at room temperature for 7 days. Finally, the tube fluid was checked for detection of larvae of Trichostrongylus species (Harada and Mori, 1955).

For the sodium chloride flotation technique or the Willis method, about 2-3 g of each fecal sample was diluted in $20 \mathrm{ml}$ of saturated salt solution $(\mathrm{NaCl}, 1.20 \mathrm{~g} / \mathrm{ml})$. The mixture was then filtered through sterile gauze and immediately transferred into a 
test tube. Then, a coverslip was placed carefully on top of the tube. After $15 \mathrm{~min}$, the coverslip was lifted off the test tube and deposited on a microscope slide (Willis, 1921).

\section{Molecular Methods DNA Extraction}

For DNA isolation, about $2 \mathrm{~g}$ of each stool sample was processed using the sodium chloride flotation technique. Next, the supernatant was washed twice with distilled water, followed by centrifugation at $8,000 \times g$ for $5 \mathrm{~min}$ to remove the salt. Subsequently, genomic DNA was extracted from the sediment using a commercial DNA extraction kit (Viragene, Tehran, Iran) based on the instructions on the manual and stored at $-20^{\circ} \mathrm{C}$ for PCR amplification.

\section{PCR Amplification}

PCR reactions were performed in $20 \mu \mathrm{l}$ volumes containing $2 \times$ red PCR premix (Ampliqon, Odense, Denmark), 20 pmol of each primer, and $3 \mu \mathrm{l}$ of extracted DNA. The ribosomal DNA internal transcribed spacer 2 (ITS2) was amplified using forward primer (Tri-F: 5'-AATGAATTTCTACAGTGTGG-3') and reverse primer (Tri-R: 5'-CATACATGTCCCTGTTTAAATC-3'), resulting in an amplicon size for Trichostrongylus spp. of 211 bp (Mizani et al., 2017). The PCR conditions comprised an initial denaturing step of $95^{\circ} \mathrm{C}$ for $5 \mathrm{~min}$, followed by 35 cycles of denaturation at $95^{\circ} \mathrm{C}$ for 45 $\mathrm{s}$, annealing at $54^{\circ} \mathrm{C}$ for $45 \mathrm{~s}$, and extension at $72^{\circ} \mathrm{C}$ for $60 \mathrm{~s}$, and a final extension at $72^{\circ} \mathrm{C}$ for $7 \mathrm{~min}$. Finally, the PCR product was electrophoresed on $1.5 \%$ agarose gel and visualized with a UV transilluminator. Later, the PCR products were sent to a domestic sequencing company (Codon Genetic Company, Tehran, Iran) for sequence determination via the Sanger method.

To confirm the results of the molecular method, eight positive products-four positive and four negative parasitological stool samples-were selected randomly and sent to a domestic sequencing company (Codon Genetic Company, Tehran, Iran) for sequence determination via the Sanger method. Sequence results were manually edited and analyzed using Chromas (version 2.6) software. The sequences were compared with those submitted to GenBank using the BLAST system (http:// www.ncbi.nlm.nih.gov/), and multiple sequence alignment was carried out using the Clustal W method of Bioedit software (version 7.2).

\section{Analytical Sensitivity and Specificity of PCR}

To determine the analytical sensitivity of the molecular method, genomic DNA was extracted from 100, 50, 20, 10, 5, and from 1 egg of Trichostrongylus spp. using a DNA extraction kit (Viragene, Tehran, Iran) according to the manufacturer's protocol. Subsequently, PCR for these samples was carried out as mentioned above.

The specificity of the PCR method was assessed using extracted DNAs from adult Necator americanus, Taenia saginata, Haemonchus contortus, Marshallagia marshalli, Ostertagia ostertagi, and Rhabditis axei, and also from stool samples infected with S. stercoralis, Fasciola hepatica, Dicrocoelium denriticum, Cryptosporidium sp., Enterocytozoon bieneusi, Entamoeba coli, Giardia lamblia, and Blastocystis hominis.

\section{Data Analysis}

Data were analyzed using SPSS software (version 18, SPSS Inc., Chicago, IL, USA) to determine the diagnostic sensitivity and specificity of the molecular and parasitological methods.

\section{Ethical Approval}

This study was reviewed and approved by the Ethics Committees of Guilan University of Medical Sciences, Iran (ref. no. IR.GUMS.REC.1398.434).

\section{RESULTS}

\section{Comparison of Parasitological and PCR Methods}

Among the total of 206 individuals examined, 72 people (35\%) were found infected with Trichostrongylus species using combined parasitological methods (Figure 1). The detection rates for the wet mount, FEAC, APC, HMC, and Willis techniques solely were 4 (1.9\%), 27 (13.1\%), 38 (18.4\%), 29 (14.1\%), and $66(32 \%)$, respectively (Table 1). The Willis method, as the most sensitive among the parasitological methods, could detect 4 of the 4 positive cases found using wet mount, 26 of the 27 by FEAC, 27 of the 29 by HMC, and 35 of the 38 by APC. In addition, the Willis technique detected 62, 40, 39, and 31 more samples, which were negative by wet mount, FEAC, $\mathrm{HMC}$, and APC, respectively. Moreover, the other intestinal parasites detected in the current study using parasitological methods were S. stercoralis $(0.97 \%)$, G. lamblia $(1.4 \%), B$. hominis (0.97\%), and F. hepatica (0.48\%).

The PCR method detected Trichostrongylus species DNA in 79 out of 206 stool samples (38.3\%) (Figure 2). All these positive cases were detected using wet mount, FEAC, and HMC. However, PCR failed to detect one APC and two Willis positive cases. Also, the PCR assay detected nine (4.37\%) more samples, which were negative using the parasitological methods (Table 2). Sequence analysis of $167 \mathrm{bp}$ of the eight randomly selected PCR products revealed that all of them had $100 \%$ similarity to $T$. colubriformis in the GenBank reference sequences. All positive isolates were registered in the GenBank database with accession numbers MW680815-MW680822.

Since there is no valid gold standard for the diagnosis of Trichostrongylus spp. infections, the combined results of the parasitological methods were considered as the gold standard in this study. Therefore, the diagnostic sensitivity values of the wet mount, HMC, Willis, APC, FEAC, and PCR methods were calculated as $5.6 \%, 40.3 \%, 91.7 \%, 52.8 \%, 37.5 \%$, and $97.2 \%$, respectively. The diagnostic specificity of all the parasitological methods was $100 \%$, while that of the PCR assay was $93.3 \%$. 


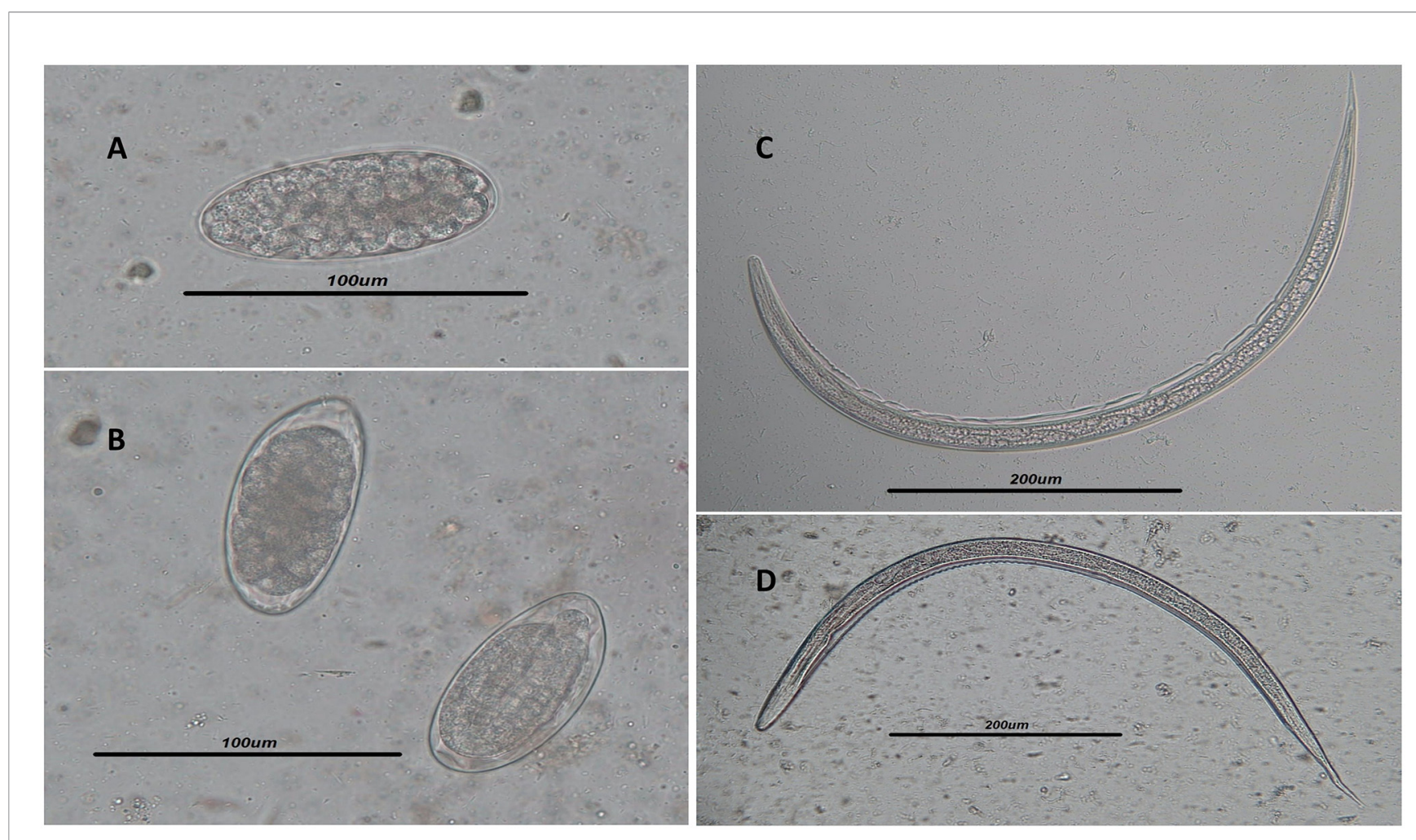

FIGURE 1 | (A, B) Light microscope view of Trichostrongylus spp. eggs isolated by the Willis technique in the stool samples of infected humans. Scale bar, $100 \mu$ m. (C, D) Filariform larvae of Trichostrongylus spp. isolated from agar plate culture. Scale bar, $200 \mu \mathrm{m}$.

\section{Analytical Sensitivity and Specificity of PCR}

The detection limit for the PCR method was DNA of one Trichostrongylus spp. egg (Figure 3). No amplification was found in the PCR assay from the DNAs extracted from all the above-mentioned intestinal parasites, except Trichostrongylus species. The results illustrated that the PCR assay was highly specific for the detection of Trichostrongylus spp.

\section{DISCUSSION}

Recent epidemiological studies have shown that the prevalence of most human STHs, such as Ascaris lumbricoides and hookworms, has decreased sharply in Iran; however, S. stercoralis and Trichostrongylus species are still being reported in a few parts of the country (Rokni, 2008; Sharifdini et al., 2017a; Sharifdini et al., 2017b; Sharifdini et al., 2020). Utilizing an accurate diagnostic method is one of the most important tools in the effective prevention and control of human trichostrongylosis. Our findings provide important new information on the performance of five types of parasitological methods and the PCR assay for the diagnosis of Trichostrongylus infection in humans.

In this study, the Willis technique detected Trichostrongylus eggs in 66 of the 206 stool samples with a sensitivity of $91.7 \%$ and a specificity of $100 \%$. The sensitivity of the Willis technique for Trichostrongylus diagnosis was considerably much higher compared with that of the other parasitological methods. This method diagnosed $100 \%, 96.3 \%, 93.1 \%$, and $92.1 \%$ of Trichostrongylus cases detected using the wet mount, FEAC, HMC, and APC methods, respectively. Also, the Willis technique detected Trichostrongylus eggs in 62, 40, 39, and 31 samples, which were scored as negative by wet mount, FEAC, HMC, and APC, respectively. Our results confirmed previous

TABLE 1 | Comparison of the parasitological and molecular methods in the detection of Trichostrongylus spp. in fecal samples $(n=206)$.

\begin{tabular}{|c|c|c|c|c|c|c|}
\hline & Wet mount & FEAC & APC & HMC & Willis & PCR \\
\hline Negative (134) & 202 & 179 & 168 & 177 & 140 & 127 \\
\hline Specificity (\%) & 100 & 100 & 100 & 100 & 100 & 93.3 \\
\hline
\end{tabular}

FEAC, formalin ethyl acetate concentration; APC, agar plate culture; HMC, Harada-Mori culture. 


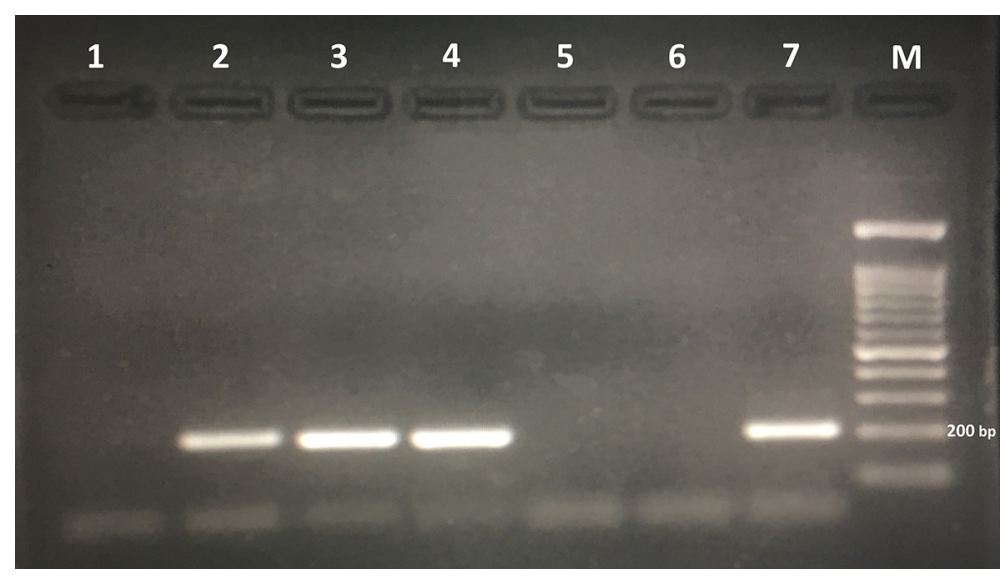

FIGURE 2 | Agarose gel electrophoresis of polymerase chain reaction products amplified with genomic DNA from stool samples. Lanes 2, 3, and 4: polymerase chain reaction products of stool samples positive for Trichostrongylus spp. Lane 6: negative control. Lane 7: positive control (Trichostrongylus colubriformis). M, 100bp DNA marker.

findings that the fecal flotation technique is a highly sensitive diagnostic test for STHs, especially hookworms (Inpankaew et al., 2014; Clarke et al., 2018; Zeleke et al., 2020). On the other hand, this technique is simple and faster, cheaper, and is user-friendly compared to the FEAC, HMC, and APC methods for the diagnosis of trichostrongylosis. Zeleke et al. reported that both the sensitivity and diagnostic accuracy of the fecal flotation technique were $100 \%$ for the detection of hookworm infections (Zeleke et al., 2020).

The present study also demonstrated that APC was the second most sensitive parasitological examination, with a sensitivity of $52.8 \%$. Although several studies have confirmed APC as being more sensitive than the other parasitological tests in the diagnosis of S. stercoralis infection (Sharifdini et al., 2014; Sharifdini et al., 2015; Mirzaei et al., 2021), its sensitivity was much less than that of the Willis technique for the detection of Trichostrongylus eggs in the current study. Additionally, this method is time-consuming, labor-intensive, and requires a well-trained microscopist (Ericsson et al., 2001). In the current study, HMC and FEAC were ranked as the third (40.3\%) and fourth (37.5\%) most sensitive techniques, respectively. Until now, only two studies have been performed comparing parasitological methods such as APC and FEAC for the diagnosis of human trichostrongylosis. Similar to our results, Najmi et al. reported that APC (88.23\%) was more sensitive than FEAC $(62.75 \%)$ in the diagnosis of human trichostrongyliasis (Najmi et al., 2017). In contrast to our findings, another study showed that the sensitivity of FEAC for the detection of Trichostrongylus was higher than that of APC (95.8\% vs. 90.1\%) (Saraei et al., 2019). The observed differences in the sensitivity between the two methods in these studies could be due to the skill variations of technicians.

Our study findings illustrated that the direct wet mount method had very low sensitivity $(5.6 \%)$ for the diagnosis of trichostrongylosis compared to the other parasitological methods. This is similar to other studies, which demonstrated that the direct wet mount method had a low detection ability for intestinal helminthic infections and may lead to false-negative results (Mengist et al., 2018; Demeke et al., 2021).

PCR-based techniques using the ITS2 region of rDNA are considered effective tools for the detection and identification of Trichostrongylus species in human fecal samples (Gholami et al., 2015; Sharifdini et al., 2017c; Perandin et al., 2018; Hidalgo et al., 2020). The DNA isolation procedure is a critical step that is helpful in the efficacy of molecular methods. In this study, similar to that in others (Hidalgo et al., 2018; Hidalgo et al., 2020), the processing of stool samples using the flotation technique was applied efficiently for DNA isolation. This method significantly reduced the PCR inhibitory substances in the stool samples, such as bacterial proteases, nucleases, cell debris, and bile acids, and resulted in the improved detection rate of PCR. On the other hand, this isolation method is rapid, labor-effective, and can be applied in the detection of light-intensity infections.

TABLE 2 | Evaluation of the PCR assay in comparison with parasitological methods for the diagnosis of Trichostrongylus spp. infection in stool samples ( $n=206)$.

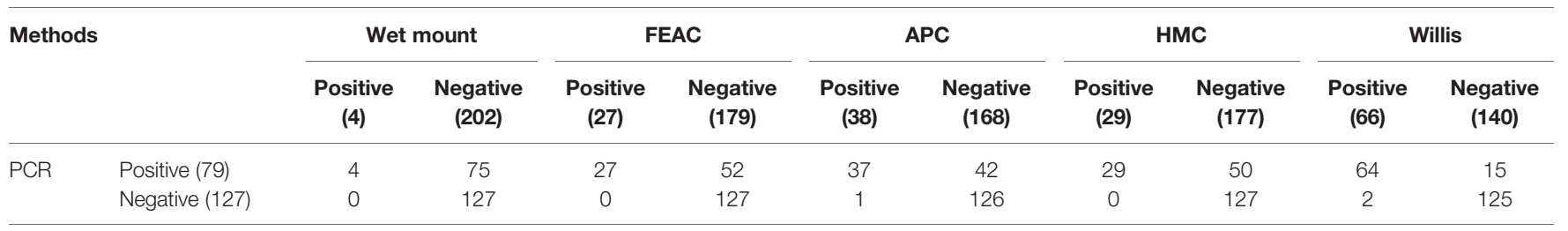

FEAC, formalin ethyl acetate concentration; APC, agar plate culture; HMC, Harada-Mori culture 


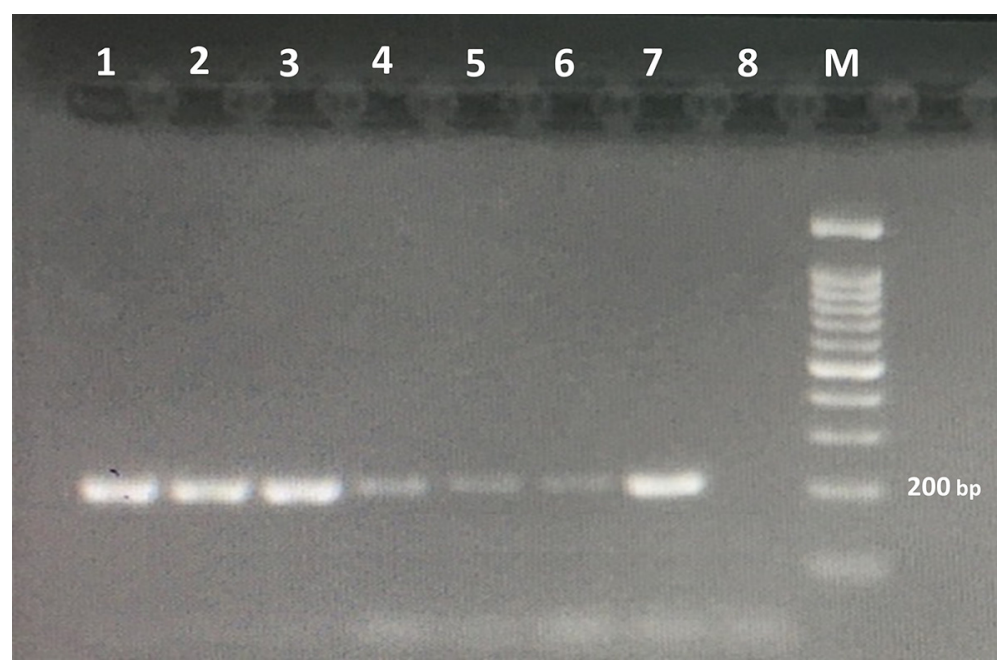

FIGURE 3 | Agarose gel electrophoresis of the PCR products amplified with genomic DNA from stool samples. The PCR products are as follows: lane 1, DNA from 100 eggs of Trichostrongylus species; lane 2, DNA from 50 eggs of Trichostrongylus species; lane 3, DNA from 20 eggs of Trichostrongylus species; lane 4, DNA from 10 eggs of Trichostrongylus species; lane 5, DNA from 5 eggs of Trichostrongylus species; lane 6, DNA from one egg of Trichostrongylus species; lane 7 , positive control (Trichostrongylus colubriformis); lane 8: negative control. M, 100-bp DNA marker.

The PCR method detected Trichostrongylus spp. DNA in 79 fecal samples with a sensitivity of $97.2 \%$ and a specificity of 93.3\%. Our findings illustrated that this method was more sensitive than are the parasitological methods in the diagnosis of Trichostrongylus species infection. It detected all samples that had been detected as positive by the wet mount, FEAC, and HMC methods, but could not detect one APC and two Willis positive cases. In addition, PCR was positive in nine samples (4.37\%) that had not been detected by the parasitological methods. This study, for the first time, compared molecular methods with various parasitological examinations for the diagnosis of human trichostrongylosis. However, several studies have shown that PCR-based methods are more sensitive than are conventional parasitological techniques for the detection of intestinal helminthic infections (Sharifdini et al., 2015; Chidambaram et al., 2017).

Our sequence analysis showed that all PCR products, including the negative and positive parasitological samples, were confirmed as T. colubriformis. Therefore, based on these negative parasitological samples that were true positives with PCR, the specificity of the PCR assay will be increased. Additionally, the sequence analysis confirmed previous studies showing that $T$. colubriformis are a predominant species in residents of Northern Iran (Gholami et al., 2015; Sharifdini et al., 2017a; Sharifdini et al., 2017c; Ashrafi et al., 2020).

This is the first study evaluating PCR in comparison to various parasitological methods for the detection of Trichostrongylus species in fecal samples. Our findings showed that, among the different parasitological methods evaluated, the Willis technique was more sensitive than are the others. While the PCR method is superior to the Willis technique in the detection of positive cases, the Willis technique is simple, rapid, and inexpensive, and only simple technology and equipment are required to propose screening and epidemiological studies. In addition, although PCR is an expensive method, it is not dependent on skilled microscopists and is feasible in detecting infections with low parasite numbers.

\section{DATA AVAILABILITY STATEMENT}

The datasets presented in this study can be found in online repositories. The names of the repository/repositories and accession number(s) can be found below: NCBI [accession: MW680815-MW680822].

\section{ETHICS STATEMENT}

The studies involving human participants were reviewed and approved by Ref. No. IR.GUMS.REC.1398.434. The patients/ participants provided their written informed consent to participate in this study.

\section{AUTHOR CONTRIBUTIONS}

MS designed the study. MP collected the samples. MP and BR carried out the parasitological methods. MP, MS, and NH performed the molecular method. MS, ZR, and KA analyzed the data. MS drafted the manuscript. All authors read and approved the final version of the manuscript. 


\section{FUNDING}

This study has been fnancially supported by Research Deputy of Guilan University of Medical Sciences, with project No. 98072002.

\section{ACKNOWLEDGMENTS}

The authors would like to thank all those who have contributed to this research.

\section{REFERENCES}

Ashrafi, K., Sharifdini, M., Heidari, Z., Rahmati, B., and Kia, E. B. (2020). Zoonotic Transmission of Teladorsagia Circumcincta and Trichostrongylus Species in Guilan Province, Northern Iran: Molecular and Morphological Characterizations. BMC Infect. Dis. 20, 28. doi: 10.1186/s12879-020-4762-0

Ashrafi, K., Tahbaz, A., Sharifdini, M., and Mas-Coma, S. (2015). Familial Trichostrongylus Infection Misdiagnosed as Acute Fascioliasis. Emerg. Infect. Dis. 21, 1869-1870. doi: 10.3201/eid2110.141392

Boreham, R. E., Mccowan, M. J., Ryan, A. E., Allworth, A. M., and Robson, J. M. (1995). Human Trichostrongyliasis in Queensland. Pathology 27, 182-185. doi: 10.1080/00313029500169842

Cancrini, G., Boemi, G., Iori, A., and Corselli, A. (1982). Human Infestations by Trichostrongylus axei, T. capricola and T. vitrinus: 1st Report in Italy. Parassitologia 24, 145-149.

Chidambaram, M., Parija, S. C., Toi, P. C., Mandal, J., Sankaramoorthy, D., George, S., et al. (2017). Evaluation of the Utility of Conventional Polymerase Chain Reaction for Detection and Species Differentiation in Human Hookworm Infections. Trop. Parasitol. 7, 111-117. doi: 10.4103/tp.TP_26_17

Clarke, N. E., Llewellyn, S., Traub, R. J., Mccarthy, J., Richardson, A., and Nery, S. V. (2018). Quantitative Polymerase Chain Reaction for Diagnosis of SoilTransmitted Helminth Infections: A Comparison With a Flotation-Based Technique and an Investigation of Variability in DNA Detection. Am. J. Trop. Med. Hyg. 99, 1033. doi: 10.4269/ajtmh.18-0356

Demeke, G., Fenta, A., and Dilnessa, T. (2021). Evaluation of Wet Mount and Concentration Techniques of Stool Examination for Intestinal Parasites Identification at Debre Markos Comprehensive Specialized Hospital, Ethiopia. Infect. Drug Resistance 14, 1357-1362. doi: 10.2147/IDR.S307683

Ericsson, C. D., Steffen, R., Siddiqui, A. A., and Berk, S. L. (2001). Diagnosis of Strongyloides stercoralis Infection. Clin. Infect. Dis. 33, 1040-1047. doi: $10.1086 / 322707$

Ghadirian, E. (1977). Human Infection With Trichostrongylus lerouxi (Biocca, Chabaud, and Ghadirian 1974) in Iran. Am. J. Trop. Med. Hyg. 26, 1212-1213. doi: 10.4269/ajtmh.1977.26.1212

Ghadirian, E., and Arfaa, F. (1975). Present Status of Trichostrongyliasis in Iran. Am. J. Trop. Med. Hyg. 24, 935-941. doi: 10.4269/ajtmh.1975.24.935

Ghadirian, E., Arfaa, F., and Sadighian, A. (1974). Human Infection With Trichostrongylus capricola in Iran. Am. J. Trop. Med. Hyg. 23, 1002-1003. doi: 10.4269/ajtmh.1974.23.1002

Ghanbarzadeh, L., Saraei, M., Kia, E. B., Amini, F., and Sharifdini, M. (2019). Clinical and Haematological Characteristics of Human Trichostrongyliasis. J. Helminthol. 93, 149-153. doi: 10.1017/S0022149X17001225

Gholami, S., Babamahmoodi, F., Abedian, R., Sharif, M., Shahbazi, A., Pagheh, A., et al. (2015). Trichostrongylus colubriformis: Possible Most Common Cause of Human Infection in Mazandaran Province, North of Iran. Iran J. Parasitol. 10, 110-115.

Harada, Y., and Mori, O. (1955). A New Method for Culturing Hook Worm. Yonago Acta Med. 1, 177-179.

Hidalgo, A., Gacitúa, P., Melo, A., Oberg, C., Herrera, C., and Fonseca-Salamanca, F. (2020). First Molecular Characterization of Trichostrongylus colubriformis Infection in Rural Patients From Chile. Acta Parasitol. 65, 790-795. doi: 10.2478/s11686-020-00206-1

Hidalgo, A., Melo, A., Romero, F., Hidalgo, V., Villanueva, J., and FonsecaSalamanca, F. (2018). DNA Extraction in Echinococcus granulosus and Taenia Spp. Eggs in Dogs Stool Samples Applying Thermal Shock. Exp. Parasitol. 186, 10-16. doi: 10.1016/j.exppara.2018.01.016

Inpankaew, T., Schär, F., Khieu, V., Muth, S., Dalsgaard, A., Marti, H., et al. (2014). Simple Fecal Flotation is a Superior Alternative to Guadruple Kato Katz Smear Examination for the Detection of Hookworm Eggs in Human Stool. PloS Negl. Trop. Dis. 8, e3313. doi: 10.1371/journal.pntd.0003313
Lattes, S., Ferte, H., Delaunay, P., Depaquit, J., Vassallo, M., Vittier, M., et al. (2011). Trichostrongylus colubriformis Nematode Infections in Humans, France. Emerg. Infect. Dis. 17, 1301-1302. doi: 10.3201/eid1707.101519

Mengist, H. M., Demeke, G., Zewdie, O., and Belew, A. (2018). Diagnostic Performance of Direct Wet Mount Microscopy in Detecting Intestinal Helminths Among Pregnant Women Attending Ante-Natal Care (ANC) in East Wollega, Oromia, Ethiopia. BMC Res. Notes 11, 1-6. doi: 10.1186/s13104-018-3380-Z

Mirzaei, L., Ashrafi, K., Roushan, Z. A., Mahmoudi, M. R., Masooleh, I. S., Rahmati, B., et al. (2021). Strongyloides stercoralis and Other Intestinal Parasites in Patients Receiving Immunosuppressive Drugs in Northern Iran: A Closer Look at Risk Factors. Epidemiol. Health 43, 1-7. doi: 10.4178/epih. e2021009

Mizani, A., Gill, P., Daryani, A., Sarvi, S., Amouei, A., Katrimi, A. B., et al. (2017). A Multiplex Restriction Enzyme-PCR for Unequivocal Identification and Differentiation of Trichostrongylus Species in Human Samples. Acta Trop. 173, 180-184. doi: 10.1016/j.actatropica.2017.06.001

Najmi, B., Kia, E., Hosseini, M., Mobedi, I., and Kamranrashani, B. (2017). Comparative Efficacy of Nutrient Agar Plate Culture and Formalin Ether Concentration Methods in the Laboratory Diagnosis of Human Trichostrongyliasis. J. Guilan Univ. Med. Sci. 25, 57-65.

Perandin, F., Pomari, E., Bonizzi, C., Mistretta, M., Formenti, F., and Bisoffi, Z. (2018). Assessment of Real-Time Polymerase Chain Reaction for the Detection of Trichostrongylus spp. DNA From Human Fecal Samples. Am. J. Trop. Med. Hyg. 98, 768. doi: 10.4269/ajtmh.17-0733

Phosuk, I., Intapan, P. M., Sanpool, O., Janwan, P., Thanchomnang, T., Sawanyawisuth, K., et al. (2013). Molecular Evidence of Trichostrongylus colubriformis and Trichostrongylus axei Infections in Humans From Thailand and Lao PDR. Am. J. Trop. Med. Hyg. 89, 376-379. doi: 10.4269/ajtmh.13-0113

Roberts, L. S., and Janovy, J. (2012). Foundations of Parasitology (New York: McGraw-Hill)

Rokni, M. (2008). The Present Status of Human Helminthic Diseases in Iran. Ann. Trop. Med. Parasitol. 102, 283-295. doi: 10.1179/136485908X300805

Saraei, M., Ghanbarzadeh, L., Hajialilo, E., Barghandan, T., Amini, F., and Sharifdini, M. (2019). Comparison of Nutrient Agar Plate Culture and Formalin-Ethyl Acetate Concentration Methods in Diagnosis of Human Trichostrongyliasis. J. Ardabil. Univ. Med. Sci. 18, 506-514. doi: 10.29252/ jarums.18.4.506

Sato, M., Yoonuan, T., Sanguankiat, S., Nuamtanong, S., Pongvongsa, T., Phimmayoi, I., et al. (2011). Short Report: Human Trichostrongylus colubriformis Infection in a Rural Village in Laos. Am. J. Trop. Med. Hyg. 84, 52-54. doi: 10.4269/ajtmh.2011.10-0385

Sharifdini, M., Derakhshani, S., Alizadeh, S. A., Ghanbarzadeh, L., Mirjalali, H., Mobedi, I., et al. (2017a). Molecular Identification and Phylogenetic Analysis of Human Trichostrongylus Species From an Endemic Area of Iran. Acta Trop. 176, 293-299. doi: 10.1016/j.actatropica.2017.07.001

Sharifdini, M., Eshrat Beigom, K., Ashrafi, K., Hosseini, M., Mirhendi, H., Mohebali, M., et al. (2014). An Analysis of Clinical Characteristics of Strongyloides stercoralis in 70 Indigenous Patients in Iran. Iranian J. Parasitol. 9, 155.

Sharifdini, M., Ghanbarzadeh, L., Barikani, A., and Saraei, M. (2020). Prevalence of Intestinal Parasites Among Rural Inhabitants of Fouman, Guilan Province, Northern Iran With Emphasis on Strongyloides Stercoralis. Iran J. Parasitol. 15, 91-100. doi: 10.18502/ijpa.v15i1.2531

Sharifdini, M., Ghanbarzadeh, L., Kouhestani-Maklavani, N., Mirjalali, H., and Saraei, M. (2017b). Prevalence and Molecular Aspects of Human Hookworms in Guilan Province, Northern Iran. Iranian J. Parasitol. 12, 374.

Sharifdini, M., Heidari, Z., Hesari, Z., Vatandoost, S., and Kia, E. B. (2017c). Molecular Phylogenetics of Trichostrongylus Species (Nematoda: Trichostrongylidae) From Humans of Mazandaran Province, Iran. Korean J. Parasitol. 55, 279-285. doi: 10.3347/kjp.2017.55.3.279 
Sharifdini, M., Mirhendi, H., Ashrafi, K., Hosseini, M., Mohebali, M., Khodadadi, H., et al. (2015). Comparison of Nested Polymerase Chain Reaction and RealTime Polymerase Chain Reaction With Parasitological Methods for Detection of Strongyloides stercoralis in Human Fecal Samples. Am. J. Trop. Med. Hyg. 93, 1285-1291. doi: 10.4269/ajtmh.15-0309

Wall, E. C., Bhatnagar, N., Watson, J., and Doherty, T. (2011). An Unusual Case of Hypereosinophilia and Abdominal Pain: An Outbreak of Trichostrongylus Imported From New Zealand. J. Travel Med. 18, 59-60. doi: 10.1111/j.17088305.2010.00474.x

Watthanakulpanich, D., Pongvongsa, T., Sanguankiat, S., Nuamtanong, S., Maipanich, W., Yoonuan, T., et al. (2013). Prevalence and Clinical Aspects of Human Trichostrongylus colubriformis Infection in Lao PDR. Acta Trop. 126, 37-42. doi: 10.1016/j.actatropica.2013.01.002

Willis, H. H. (1921). A Simple Levitation Method for the Detection of Hookworm Ova. Med. J. Aust. 2, 375-376. doi: 10.5694/j.1326-5377.1921.tb60654.x

Zeleke, A. J., Addisu, A., Derso, A., Tegegne, Y., Birhanie, M., and Sisay, T. (2021). Evaluation of Hookworm Diagnosis Techniques from Patients in Debre Elias and Sanja Districts of the Amhara Region, Ethiopia. J. Parasitol. Res. 1-7 doi: $10.1155 / 2021 / 6682330$
Conflict of Interest: The authors declare that the research was conducted in the absence of any commercial or financial relationships that could be construed as a potential conflict of interest.

Publisher's Note: All claims expressed in this article are solely those of the authors and do not necessarily represent those of their affiliated organizations, or those of the publisher, the editors and the reviewers. Any product that may be evaluated in this article, or claim that may be made by its manufacturer, is not guaranteed or endorsed by the publisher.

Copyright (c) 2021 Pandi, Sharifdini, Ashrafi, Atrkar Roushan, Rahmati and Hajipour. This is an open-access article distributed under the terms of the Creative Commons Attribution License (CC BY). The use, distribution or reproduction in other forums is permitted, provided the original author(s) and the copyright owner(s) are credited and that the original publication in this journal is cited, in accordance with accepted academic practice. No use, distribution or reproduction is permitted which does not comply with these terms. 